\title{
Confiabilidade intra-avaliador da medida de amplitude de movimento da flexão e extensão do joelho pelo método de fotogrametria
}

\author{
Intra-rater reliability of knee flexion and extension range of \\ motion measurement through the photogrammetry method \\ Eurico Peixoto César ${ }^{1,2,3,4}$, Paulo Sergio Chagas Gomes ${ }^{2,3,5}$, Caroline Lopes Marques ${ }^{3}$, \\ Bruno D’Ávila Palmeira Domingos ${ }^{3}$, Tony Meireles dos Santos ${ }^{2,3,5}$
}

RESUMO I Apesar de existirem diversos métodos para a determinação da amplitude de movimento (ADM), a baixa confiabilidade, a pouca sensibilidade, a subjetividade ou a ausência de valores do erro típico da medida (ETM) de alguns desses métodos comprometem a interpretação adequada dos resultados. O objetivo deste estudo foi determinar a confiabilidade intra-avaliador da medida e do método de fotogrametria para a ADM de extensão ativa e flexão passiva do joelho (FPJ), realizada no mesmo dia (consistência interna) e em dias diferentes (estabilidade). Participaram 18 sujeitos do sexo masculino (24,5 $\pm 3,7$ anos,

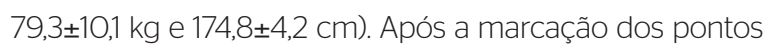
anatômicos de referência, a angulação dos movimentos foi registrada em fotografia, por meio da ferramenta de dimensão angular no software CorelDRAW ${ }^{\circledR}$. A confiabilidade da medida e do instrumento foi estabelecida pelo coeficiente de correlação intraclasse (CCl) e pelo cálculo do ETM. A distribuição dos erros dos dados foi verificada pela representação gráfica de Bland e Altman. O método apresentou confiabilidade perfeita ( $E T M=0,01$ e $C C l=1,0$ ) para ambos os movimentos. Para a confiabilidade da medida, foram encontrados os valores 0,97 e 0,93 para consistência interna e 0,96 e 0,83 para estabilidade (ETM=2,9 e 4,0\%; $\mathrm{p}<0,001)$ na extensão e flexão do joelho, respectivamente.

\begin{abstract}
I Although there are several methods for determining the range of motion (ROM), low reliability, low sensitivity, subjectivity or lack of information related to the typical error of measurement (TEM) may compromise interpretation of testing results. The aim of this study was to test the intra-rater reliability of the ROM measurement and the photogrammetry method for same day (internal consistency) and between days (stability) for knee active extension and passive flexion. Eighteen active male volunteers ( $24.5 \pm 3.7$ years, $79.3 \pm 10.1 \mathrm{~kg}$ and $174.8 \pm 4.2 \mathrm{~cm}$ ) were selected to participate in the study. After identification of anatomical landmarks used for reference, knee passive flexion and active extension were recorded by photography and then analyzed using an angular dimension tool from CoreIDRAW ${ }^{\circledR}$. The reliability of the measurement and the instrument was determined using intraclass correlation coefficient (ICC) and TEM. Data distribution was observed using the graphical representation of Bland e Altman. The method showed a perfect reliability (TEM=0.01 and ICC=1.0) for both extension and flexion movements. The measurement showed high reliability 0.97 and 0.93 (internal consistency) and 0.96 and 0.83 (stability) (TEM=2.9 and 4.0\%, $\mathrm{p}<0.001)$ for knee extension and flexion, respectively. The method showed to be very reliable with a low typical error
\end{abstract}

Estudo desenvolvido no Laboratório Crossbridges da Universidade Gama Filho (UGF) - Rio de Janeiro (RJ), Brasil.

'Aluno do Programa de Pós-graduação (Doutorado) em Ciências do Exercício e do Esporte da UGF - Rio de Janeiro (RJ), Brasil.

²aboratório Crossbridges - UGF - Rio de Janeiro (RJ), Brasil.

Laboratório Performance - UGF - Rio de Janeiro (RJ), Brasil.

${ }^{4}$ Coordenador do Curso de Educação Física da Universidade Presidente Antônio Carlos (UNIPAC) - Barbacena (MG), Brasil.

${ }^{5}$ Docente do Programa de Pós-graduação (Doutorado) em Ciências do Exercício e do Esporte da UGF - Rio de Janeiro (RJ), Brasil. 
O método mostrou excelente confiabilidade e baixo erro na determinação da ADM de extensão e flexão do joelho, sendo assim adequado para a aplicação em dimensão clínica e de pesquisa. Descritores I amplitude de movimento articular; maleabilidade; reprodutibilidade dos testes; joelho. of the measurement knee extension and flexion ROM, showing to be adequate for application in both, clinical and research settings. Keywords I range of motion, articular; pliability; reproducibility of results; knee.

\section{INTRODUÇÃO}

Os níveis de flexibilidade de um indivíduo têm sido associados à saúde, à qualidade de vida e ao desempenho físico e esportivo ${ }^{1,2}$. Diante disso, a mensuração adequada e precisa da amplitude de movimento (ADM) é fundamental para o diagnóstico, planejamento e acompanhamento da evolução de um programa de treinamento ou tratamento ${ }^{3}$. Alguns autores apontam a relação entre flexibilidade e $\mathrm{ADM}^{4-6}$ e, diante disso, inúmeros testes clínicos foram propostos para essa medida. Dentre as estratégias propostas para a quantificação da ADM, encontram-se os testes lineares de sentar e alcançar ${ }^{7,8}$, os adimensionais, como o flexiteste ${ }^{8,9}$ e os testes angulares, como a goniometria ${ }^{10,11}$.

$\mathrm{Na}$ seleção de um determinado teste, é importante considerar sua confiabilidade, tanto em repetidas medidas em um mesmo dia quanto em dias diferentes, o que foi definido por Atikinson e Nevill como consistência interna e estabilidade, respectivamente ${ }^{12}$. Esse conceito aplica-se também ao instrumento de medida.

Dentre as diversas estratégias para a investigação da confiabilidade de um procedimento ou instrumento, o cálculo do erro típico da medida (ETM), proposto por Hopkins ${ }^{13}$, possibilita a identificação da magnitude do erro de uma medida ou equipamento. Por consequência, o efeito real decorrente da intervenção realizada pode ser adequadamente interpretado. Em geral, todos os testes clínicos citados para aferir a ADM apresentam alguma limitação, seja nos níveis de sensibilidade às pequenas variações da medida, seja na determinação do ETM $^{13}$ ou, ainda, na capacidade de alcançar valores altos de confiabilidade, tanto da medida quanto do método empregado.

Com a evolução tecnológica, novos recursos para a medida da ADM têm sido propostos. Dentre eles, destaca-se a fotogrametria digital, que, segundo a Sociedade Americana de Fotogrametria e Sensoriamento Remoto, é definida como um recurso tecnológico confiável para a obtenção de informações sobre objetos físicos por meio de gravação, medição e interpretação de imagens fotográficas ${ }^{14}$. Tal técnica possibilita o registro de mudanças sutis e da inter-relação entre partes diferentes do corpo humano, usualmente difíceis de serem mensuradas ou registradas por outros meios, facilitando a quantificação da ADM e fornecendo dados mais precisos do que os obtidos pela observação visual ${ }^{15}$.

Diante da alta praticidade de utilização da fotogrametria, seja no ambiente clínico ou esportivo e em função da carência de testes que combinem elevada sensibilidade da medida e do instrumento, em especial com informações detalhadas sobre a precisão da medida do coeficiente de correlação intraclasse (CIC) e ETM, o presente estudo objetivou verificar: a) a confiabilidade intra-avaliador do método de fotogrametria para a medida angular do arco de movimento de flexão e extensão do joelho; b) a confiabilidade da medida da amplitude máxima de movimento na flexão e extensão do joelho.

\section{MÉTODO}

\section{Sujeitos}

Participaram do estudo 18 sujeitos do sexo masculino $(24,5 \pm 3,7$ anos, $79,3 \pm 10,1 \mathrm{~kg}$ e $174,8 \pm 4,2 \mathrm{~cm})$, fisicamente ativos ( $\geq 120 \mathrm{~min} \times \mathrm{sem}^{-1}$ de atividade física), e que não faziam uso regular de qualquer recurso ergogênico nutricional e/ou farmacológico. Optou-se pela utilização de indivíduos apenas do sexo masculino com intuito de restringir a variabilidade da amostra, além da dificuldade de se controlar o efeito do ciclo menstrual das mulheres, fato que sabidamente pode influenciar indiretamente os níveis de flexibilidade ${ }^{16-18}$. Foram excluídos do estudo os sujeitos treinados em flexibilidade há, pelo menos, três meses, com história recente de lesão osteomioarticular, com segmento de coxa longo (distância entre os pontos trocanterium e tibiale laterale superiores a $50 \mathrm{~cm}$ ), em função de enquadramento adequado nas fotos, além de alguma outra contraindicação à prática de exercícios físicos. $\mathrm{O}$ presente estudo 
foi aprovado pelo Comitê de Ética em Pesquisa sob o no 0011.0.312.000-09 da Universidade Gama Filho (UGF) - Rio de Janeiro (RJ), Brasil.

\section{Delineamento experimental}

Os sujeitos realizaram três visitas experimentais, com intervalo mínimo de três e máximo de sete dias entre elas. Na primeira visita, foi realizada a familiarização dos sujeitos aos procedimentos experimentais. Nas duas visitas posteriores, em ordem determinada por sorteio, os sujeitos foram submetidos aos testes de ADM de flexão passiva do joelho (FPJ) e extensão ativa do joelho (EAJ), para posterior determinação da consistência interna e estabilidade da medida. $\mathrm{O}$ intervalo entre os procedimentos foi de $\approx 3 \mathrm{~min}$, referente ao tempo necessário para a preparação do equipamento destinado à medida seguinte e de 15 min entre os testes realizados no mesmo dia.

O melhor desempenho para cada movimento foi comparado entre os dias para a determinação da confiabilidade da medida. Para a determinação da confiabilidade do método, um único avaliador, aluno de iniciação científica, analisou as ADMs de três fotos de cada sujeito (60 no total), codificadas e organizadas aleatoriamente por um segundo pesquisador, mestrando da mesma instituição. $\mathrm{O}$ intervalo entre as avaliações foi de no mínimo três e no máximo sete dias.

\section{Procedimentos}

\section{Marcação dos pontos e preparação dos sujeitos}

Inicialmente foram identificados os pontos anatômicos Trochanterion, Tibiale Laterale e Sphyrion Laterale no membro inferior direito dos sujeitos ${ }^{19}$. Os pontos foram marcados e retocados com tinta henna para sua permanência ao longo das visitas subsequentes, garantindo assim a menor probabilidade de erro aleatório devido à inconsistência da marcação dos pontos ${ }^{13}$. Antes da medida da $\mathrm{ADM}$, os sujeitos foram orientados a permanecer sentados em repouso em uma maca durante $15 \mathrm{mi}-$ nutos. Os testes foram realizados tentando-se manter sempre o mesmo horário e temperatura $\left(\approx 23^{\circ} \mathrm{C}\right)$, a fim de evitar interferências na medida.

\section{Medida de flexão passiva do joelho}

Os sujeitos foram posicionados na maca em decúbito ventral com os joelhos estendidos e o quadril estabilizado por uma faixa com velcro. Foi realizada uma hiperextensão do quadril a aproximadamente $35^{\circ}$, apoiado em um aparato acolchoado com regulagem de altura acoplado à maca. Para o alcance de um ângulo de $35^{\circ}$, foi utilizada a lei trigonométrica dos senos para o ajuste da altura do aparato que apoiaria a coxa do avaliado, calculada considerando o seno de $35^{\circ}(0,5736)$ e a hipotenusa como o comprimento do fêmur (distância entre os pontos Trochanterion e Tibiale Laterale) estabelecida por um antropômetro (GPM-Antropômetro, Siber \& Hegner, Zurich, Suiça). Optou-se pela hiperextensão do membro para observar o real efeito do alongamento, uma vez que a maioria dos sujeitos era capaz de tocar facilmente o calcanhar no glúteo sem a adoção dessa estratégia.

Após a acomodação do sujeito, o pesquisador efetuou três movimentos passivos de flexão da articulação do joelho com o tornozelo em dorsiflexão, com um intervalo de 10 segundos entre cada repetição. A mobilização foi conduzida de forma lenta e gradual, visando o contato do calcâneo com o glúteo em um plano sagital (Figura 1A). Para que não ocorresse a abdução do quadril durante a
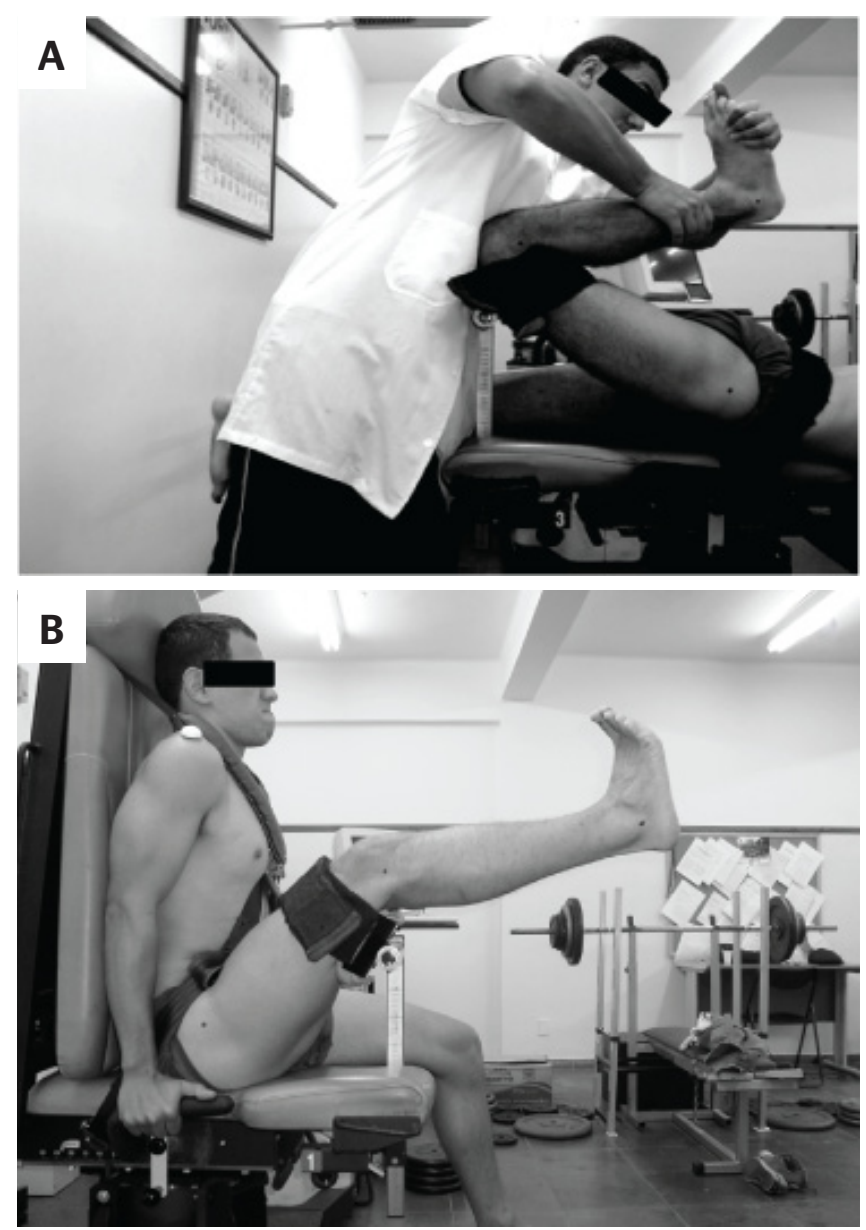

Figura 1. (A) posição final da flexão e (B) posição final de extensão de joelho 
mobilização do segmento, o avaliador fez uma obstrução manual na face lateral da coxa enquanto realizava o movimento de flexão do joelho. Foi solicitado ao sujeito que indicasse ao pesquisador quando o ponto de maior desconforto suportável fosse atingido. Essa posição era mantida por 2 segundos para que o registro fotográfico pudesse ser realizado por outro avaliador.

\section{Medida de extensão ativa do joelho}

O sujeito foi posicionado sentado em uma cadeira regulável, com o tronco totalmente apoiado e fixado no encosto em ângulo de $90^{\circ}$ com o assento, através de uma faixa com velcro passada na altura do abdômen, além de um cinto de fixação que cruzou bilateralmente o ombro e a crista ilíaca, garantindo, dessa forma, uma angulação de $90^{\circ}$ entre o tronco e o membro inferior esquerdo. $\mathrm{O}$ membro inferior direito foi apoiado na altura da região poplítea em um aparato acolchoado com regulagem de altura, garantindo uma angulação de $125^{\circ}$ de flexão do quadril com o joelho flexionado (Figura 1B). Uma faixa com velcro foi atada ao terço distal da coxa do membro testado, assegurando a estabilização dessa região durante o procedimento de testagem e a visibilidade dos pontos anatômicos marcados. A partir dessa posição, o indivíduo foi orientado a manter a articulação do tornozelo em dorsiflexão e realizar uma EAJ, de forma lenta e gradual até o ponto de maior desconforto suportado, momento em que a fotografia era obtida.

\section{Registro e análise fotográfica}

O registro fotográfico foi realizado a partir de uma câmera digital (Sony Cyber-shot ${ }^{\circledR}$ DSC-W130, 8.1 MP, zoom óptico 4x, lentes Carl Zeiss ${ }^{\circledR}$, visor LCD 2.5”, Tokyo, Japão) posicionada em um tripé, estando fixada a uma altura de um metro do solo e a uma distância de 1,5 metros do sujeito testado, de forma que o enquadramento da fotografia estivesse centralizado no ponto médio da sua coxa, com visão no plano sagital. Durante as medidas, os avaliadores se certificaram da visibilidade dos três pontos anatômicos marcados. A medida angular foi determinada por meio da ferramenta de dimensão angular do software CorelDRAW ${ }^{\circledR}$ (Corel Draw Graphics Suite, Versão 12.0, Corel Corporation, Canadá).

\section{Análise estatística}

Realizou-se a análise descritiva (média e desvio-padrão), para a caracterização da amostra. Para verificar a confiabilidade absoluta e relativa do método, utilizou-se o CCI, com classificação segundo o ponto de corte sugerido por Vincent $\mathrm{t}^{20}$ e ETM absoluto e relativo seguindo as recomendações de Hopkins ${ }^{13}$, estabelecendo-se a razão entre o desvio-padrão das diferenças entre os pares de medidas e a raiz quadrada de dois.

Para a confiabilidade da medida, foram estabelecidas a consistência interna e a estabilidade. $\mathrm{Na}$ determinação da estabilidade, foram comparadas as médias das primeiras medidas do segundo e terceiro dias. Para a determinação da consistência interna, foram comparadas a média das primeiras medidas do primeiro dia com a média das outras medidas do mesmo dia. A análise estatística utilizada foi a mesma já descrita para a confiabilidade do método (CCI e ETM). Complementarmente, foi verificado se havia relação entre a magnitude do erro e a magnitude da medida (característica homocedástica) dos dados, utilizando-se a correlação de Pearson entre a média das medidas e a diferença entre elas, além das representações gráficas de Bland-Altman. As análises estatísticas foram realizadas no Microsoft Office Excel (Microsoft Office Excel 2007, Microsoft Corporation, Redmond, EUA) e no software SPSS 17.0 for Windows ${ }^{\circledR}$ (SPSS Inc., Chicago, USA), adotando-se um nível de significância (de 5\%).

\section{RESULTADOS}

Os níveis de confiabilidade do método apresentaram característica perfeita $(\mathrm{CCI}=1,0)$ e um ETM desprezível $(\mathrm{ETM}=0,01)$. Os resultados da confiabilidade (CCI e ETM) para as medidas de flexão e extensão do joelho encontram-se na Tabela 1 . Observa-se que a estabilidade atingida nas medidas de FPJ foi um pouco menor quando comparada à consistência interna. Já nas medidas de EAJ, a consistência interna e estabilidade atingiram valores altos e praticamente idênticos, com $R \geq 0,96$ e $\mathrm{ETM} \leq 2 \%$.

Pode-se observar na Tabela 1 que o ETM apresentou valores menores para as medidas no mesmo dia (consistência interna), quando comparados às medidas em dias diferentes (estabilidade) em ambos os movimentos testados, com valores maiores para as medidas de flexão do joelho. Tal achado indica uma maior variação da medida no mesmo dia e no movimento passivo. Não foi observada diferença significativa entre a média e a diferença entre as medidas em todos os casos testados. Além disso, pode-se observar um comportamento 
homocedástico dos dados, sem relação da magnitude do erro com a magnitude da medida, como demonstrado na representação de Bland e Altman ${ }^{21}$ na Figura 2.

\section{DISCUSSÃO}

O presente estudo teve como primeiro objetivo testar a confiabilidade do método de fotogrametria para a medida da ADM. O primeiro achado demonstrou ser esta técnica precisa, possibilitando a adequada monitoração das alterações da ADM nos segmentos investigados. Foi observada precisão satisfatória da confiabilidade da medida tanto para flexão quanto para extensão do joelho. Em conjunto, os achados aqui reportados proporcionam informações sobre variações esperadas na medida com a utilização do teste de fotogrametria, obtidas a partir do ETM. Essas informações se fazem necessárias para a interpretação das variações obtidas após um período de intervenção com fins terapêuticos ou atléticos, aprimorando o poder de julgamento do avaliador em determinar se a mudança é real ou apenas decorrente do erro natural da própria medida.

Inúmeros estudos têm se dedicado a verificar a confiabilidade de métodos de determinação da $\mathrm{ADM}^{14,22-25}$. Por exemplo, em relação aos testes lineares, Hui e Yuen ${ }^{23}$ relatam que os diversos protocolos de sentar e alcançar produzem uma consistência interna, variando entre 0,89 e 0,98 . No entanto, esses testes apresentam problemas, como uma validade moderada $(\mathrm{r}=0,67)$ para os músculos ísquiotibiais e fraca $(\mathrm{r}=0,44)$ para músculos da coluna lombar ${ }^{26}$. Além disso, alguns autores ${ }^{7,27,28}$ apontaram a influência dos fatores antropométricos, como o comprimento desproporcional dos membros em relação ao tronco ou vice-versa, como fatores de confundimento na interpretação dos resultados ${ }^{29}$.

Sobre os testes adimensionais, alguns autores alegam que as estimativas visuais da $\mathrm{ADM}$ possuem alto teor de subjetividade, tendendo a subestimar os escores obtidos em caso de dúvida na interpretação da amplitude alcançada e que são pouco sensíveis a mudanças mais discretas, sendo inadequado para estudos ou condições em que maiores níveis de precisão da medida sejam necessários $^{22,30}$. Entretanto, já foi demonstrado nível de confiabilidade para o Flexiteste de $\mathrm{R}=0,81^{8}$.

Diversos estudos têm utilizado o método de goniometria em diferentes movimentos para medidas de ADM. Lustosa et al. ${ }^{24}$ investigaram a confiabilidade da ADM passiva do joelho com a goniometria e
Tabela 1. Confiabilidade e precisão da medida de flexão e extensão de joelho

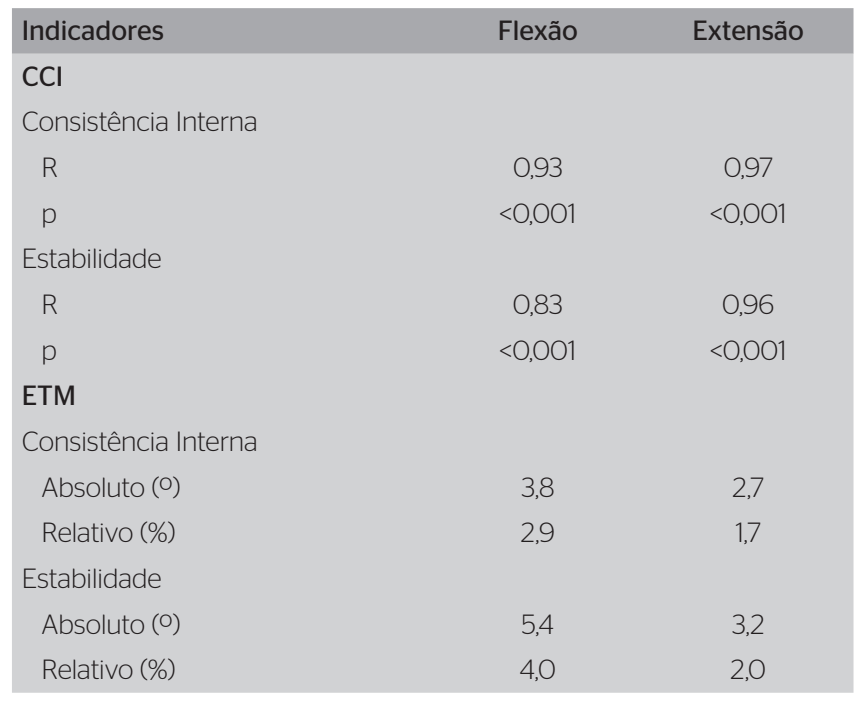

CCl: coeficiente de correlação intraclasse; R: coeficiente de correlação intraclasse; $p$ : nível de significância; ETM: erro típico da medida

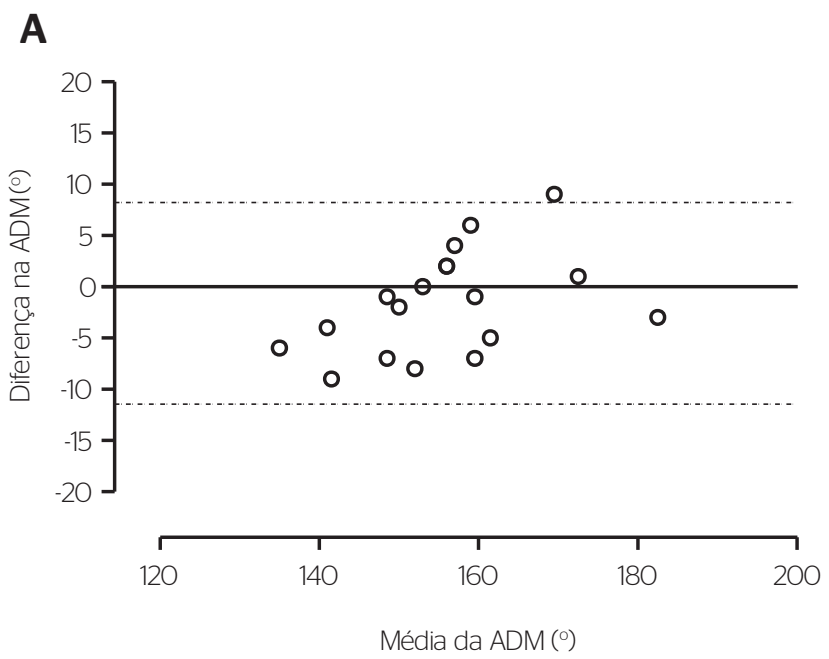

B

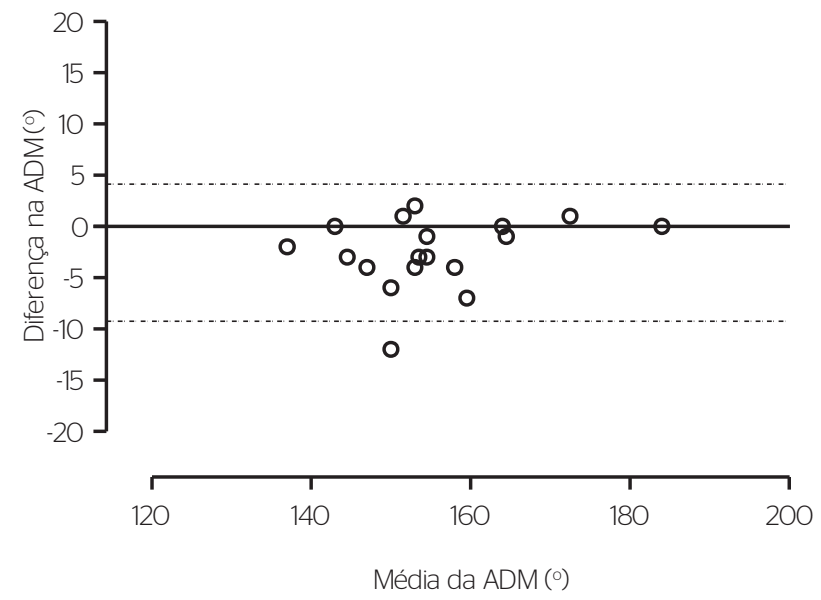

ADM: amplitude de movimento

Figura 2. Representação de Bland-Altman para confiabilidade (estabilidade) da medida de fotogrametria, nas análises de flexão (A) e extensão de joelhos (B) 
fleximetria e obtiveram CCI de 0,95 e 0,85 , respectivamente. No entanto, alguns autores relatam que esse é um método altamente dependente da proficiência no manuseio do instrumento e do conhecimento de estruturas anatômicas. Além disso, embora inúmeras pesquisas feitas sobre a confiabilidade da goniometria reportarem valores moderados a altos $(r=0,87-0,99)$, muitos estudos apontam uma melhor confiabilidade intra-avaliador do que interavaliador. Destaca-se, ainda, o fato de que alguns autores apontam uma maior confiabilidade para medidas angulares de maior magnitude ${ }^{31}$.

Comparado a esses métodos, a fotogrametria utilizada no presente estudo apresenta valores altos de consistência interna $(\mathrm{R}=0,93$ e 0,97 para flexão e extensão de joelhos, respectivamente, além da vantagem de maior facilidade de aplicação e mensuração, altos níveis de precisão e a disponibilização dos valores de ETM, o que auxilia no poder preditivo e no julgamento clínico dos resultados. Destaca-se que este é o primeiro estudo a utilizar em conjunto as determinações de CCI e ETM. Em adendo, Sacco et al. ${ }^{14}$ relatam que a fotogrametria proporciona facilidade no registro da medida de $\mathrm{ADM}$ para posterior análise, possibilitando assim mais precisão com menor interferência de variáveis de confundimento, encontrando dados mais confiáveis $(\mathrm{R}=0,97)$ do que os obtidos pela observação visual $(\mathrm{R}=0,83)$, destacando sua importância tanto do ponto de vista aplicado quanto científico.

Outra vantagem do presente método é a disponibilidade da medida real (em graus) da ADM, facilitando a sua interpretação e permitindo a percepção de alterações sutis na medida. A identificação do ETM do presente método possibilita a deteç̧ão da evolução real da ADM, sendo importante clinicamente e para o desempenho esportivo. No presente estudo, a estabilidade da medida foi classificada como Excelente $(\mathrm{R}=0,96$ para extensão joelho) e Aceitável ( $\mathrm{R}=0,83$ para flexão de joelho). Além disso, a confiabilidade do método para se medir a $A D M$ foi perfeita $(R=1,0)$, levando a concluir que o erro não se encontra no instrumento de medida e sim em outros aspectos (posicionamento do testado, percepção da amplitude articular máxima, velocidade de execução dos movimentos e a padronização da força usada na mobilização do segmento). Além disso, destaca-se a possibilidade de erros randômicos, inerente ao próprio processo de mensuração.

Futuros estudos poderão adotar a abordagem aqui sugerida, considerando a filmagem do movimento realizado ao invés do simples registro fotográfico. Essa estratégia possibilitará a identificação da velocidade de mobilização e o tempo de exposição ao estímulo de alongamento. Este estudo não investigou a confiabilidade interavaliador. Esse aspecto pode ser relevante em abordagens clínicas de grande abrangência ou multicêntrica. Além disso, estudos em outras faixas etárias, sexo e articulações necessitam ser realizados.

\section{CONCLUSÃO}

Conclui-se que a fotogrametria apresentou valores altos de confiabilidade (consistência interna e estabilidade) do método e da medida na determinação da amplitude máxima dos movimentos de FPJ e EAJ quando um único avaliador realiza as medidas, caracterizando-se como uma ferramenta sensível e com boa repetibilidade para quantificação angular dos movimentos. Em adendo, o baixo valor do ETM permite que pequenas modificações na ADM possam ser detectadas, favorecendo de maneira importante o julgamento da evolução dos níveis de flexibilidade e permitindo ao treinador ou fisioterapeuta interpretar com mais segurança os resultados decorrentes de determinada intervenção.

\section{AGRADECIMENTOS}

Agradecemos a Bruno Ribeiro Ramalho e a Renato Guerreiro pela colaboração nas análises estatísticas da confiabilidade do método. Caroline Lopes Marques recebeu apoio do PIBIC (CNPq-UGF). Tony Meireles dos Santos recebe apoio da FAPERJ e Paulo Sergio Chagas Gomes recebe apoio da FAPERJ e CNPq.

\section{REFERÊNCIAS}

1. ACSM. ACSM's Guidelines for Exercise Testing and Prescription. 8th ed. Baltimore: Lippincott Williams \& Wilkins; 2010.

2. Garber CE, Blissmer B, Deschenes MR, Franklin BA, Lamonte MJ, Lee IM, et al. American College of Sports Medicine position stand. Quantity and quality of exercise for developing and maintaining cardiorespiratory, musculoskeletal, and neuromotor fitness in apparently healthy adults: guidance for prescribing exercise. Med Sci Sports Exerc. 2011;43(7):1334-59.

3. Chagas MH, Bhering EL. Nova proposta para avaliação da flexibilidade. Rev bras Educ Fís Esp. 2004:18(3):239-48.

4. Bandy WD, Irion JM. The effect of time on static stretch on the flexibility of the hamstring muscles. Phys Ther. 1994;74(9):845-50. Discussion 50-2. 
5. Magnusson SP, Simonsen EB, Aagaard P, Boesen J, Johannsen F, Kjaer M. Determinants of musculoskeletal flexibility: viscoelastic properties, cross-sectional area, EMG and stretch tolerance. Scand J Med Sci Sports. 1997;7(4):195-202.

6. Magnusson SP, Simonsen EB, Aagaard P, Sorensen H, Kjaer M. A mechanism for altered flexibility in human skeletal muscle. J Physiol. 1996;497 (Pt 1):291-8.

7. Wells KF, Dillon EK. The sit-and-reach: a test of back and leg flexibility. Res Q Exerc Sports. 1952;23:115-8.

8. Araújo CGS. Correlação entre diferentes métodos lineares e adimensionais de avaliação da mobilidade articular. Rev Bras Ciên Mov. 2000;2:27-34.

9. Araújo CGS. Flexiteste: uma nova versão dos mapas de avaliação. Kinesis. 1986:2:251-67.

10. Norkin CC, White DJ. Measurement of joint motion: a guide to goniometry. 6th ed. Philadelphia: F. A. Davis; 1988.

11. Carregaro RL, Silva LCCB, Gil Coury HJC. Comparação entre dois testes clínicos para avaliar a flexibilidade dos músculos posteriores da coxa. Rev Bras Fisioter. 2007:11:139-45.

12. Atkinson G, Nevill AM. Statistical methods for assessing measurement error (reliability) in variables relevant to sports medicine. Sports Med. 1998:26(4):217-38

13. Hopkins WG. Measures of reliability in sports medicine and science. Sports Med. 2000;30(1):1-15.

14. Sacco ICN, Alibert S, Queiroz BWC, Pripas D, Kieling I, Kimura AA, et al. Confiabilidade da fotogrametria em relação à goniometria para avaliação postural de membros inferiores. Rev Bras Fisioter. 2007;11(5):411-7.

15. Watson AWS. Procedure for the production of high quality photographs suitable for the recording and evaluation of posture. Rev Fisioter Univ São Paulo. 1998;5(1):20-6.

16. Edward MW, Laura JH, Thomas NL, Timothy EH, Mary LVH. Association Between the Menstrual Cycle and Anterior Cruciate Ligament Injuries in Female Athletes. Am J Sports Med. 1998;26(5):614-8.

17. Tenaglia SA, McLellan TM, Klentrou PP. Influence of menstrual cycle and oral contraceptives on tolerance to uncompensable heat stress. Eur J Appl Physiol. 1999;80:76-83.

18. Chaves CPG, Simão R, Araújo CGS. Ausência de variação da flexibilidade durante o ciclo menstrual em universitárias. Rev Bras Med Esporte. 2002;8(6):212-8.
19. International Standards for Anthropometric Assessment (ISAK). 2th ed. Potchefstroom: the International society for the advancement of kinanthropometry; 2006.

20. Vincent WJ. Statistcs in kinesiology. 3th ed. Champaign, IL: Human Kinetics; 2005. p. 184.

21. Bland JM, Altman DG. Comparing methods of measurement: why plotting difference against standard method is misleading. Lancet. 1995;346(8982):1085-7.

22. Gajdosik RL, Bohannon RW. Clinical measurements of range of motion: Review of goniometry emphasizing reliability and validity. Phys Ther. 1987;67:1867-73.

23. Hui SS, Yuen PY. Validity of the modified back-saver sit-and-reach test: a comparison with other protocols. Med Sci Sports Exerc. 2000;32(9):1655-9

24. Lustosa LP, Silva CWA, Brito JP, Cordeiro RV, Lemos MS. Goniometria e fleximetria: um estudo de confiabilidade e comparação das medidas nas articulações do cotovelo e joelho. e-Scientia. 2008;1(1):1-9.

25. Patterson P, Wiksten DL, Ray L. The validity and reliability of the back saver sit-and-reach test in middle school girls and boys. Res Q Exerc Sports. 1996;67:448-51.

26. Patterson P, Wiksten DL, Ray L, Flanders C, Sanphy D. The validity and reliability of the back saver sit-and-reach test in middle school girls and boys. Res Q Exerc Sport. 1996;67(4):448-51.

27. Hoeger WW, Hopkins DR. A comparison of the sit and reach and the modified sit and reach in the measurement of flexibility in women. Res Q Exerc Sports. 1992;63:191-5.

28. Kendall FP, McCreary EK, Provance PG, Rodgers MM, Romani WA. Muscles: testing and functions. 5th ed. Baltimore, Md: Willians \& Wilkins; 2005.

29. Cornbleet SL, Woolsey NB. Assessment of hamstring muscle length in school-aged children using the sit-and-reach test and the inclinometer measure of hip joint angle. Phys Ther. 1996;76(8):850-5.

30. Lea RD. Current concepts review: range of motion measurements. J Bone Joint Surg Am. 1995;77A-8A.

31. O'Riain MD, Sibille J, Balmer S. A new goniometer for physiotherapy. Physiother Can 1983;35:279-81. 